\title{
A Study of Lead-Acid Battery Efficiency Near Top-of-Charge and the Impact on PV System Design
}

\author{
John W. Stevens and Garth P. Corey \\ Sandia National Laboratories, Photovoltaic System Applications Department \\ Sandia National Laboratories, Battery Analysis and Evaluation Department \\ PO Box 5800, MS 0753 \\ Albuquerque, New Mexico 87185-0753
}

\begin{abstract}
Knowledge of the charge efficiency of lead-acid batteries near top-of-charge is important to the design of small photovoltaic systems. In order to know how much energy is required from the photovoltaic array in order to accomplish the task of meeting load, including periodic full battery charge, a detailed knowledge of the battery charging efficiency as a function of state of charge is required, particularly in the high state-of-charge regime, as photovoltaic systems are typically designed to operate in the upper 20 to $30 \%$ of battery state-of-charge. This paper presents the results of a process for determining battery charging efficiency near top-of-charge and discusses the impact of these findings on the design of small PV systems.
\end{abstract}

\section{BACKGROUND}

Batteries are often necessary in photovoltaic (PV) systems to store energy generated while the sun is shining. Therefore, it is important to understand the specific requirements of batteries when designing a PV system. This includes an understanding of the amount of energy that will be lost in battery charging. Overestimating these battery charging losses results in a larger PV array than required, whereas underestimating them results in unanticipated loss of load as well as the possibility of damaging batteries because of lack of providing a periodic high state-of-charge.

It is generally understood that battery charge efficiency is high (above 95\%) at low states of charge and that this efficiency drops off near full charge. However, actual battery charge efficiencies are often stated as though efficiency is linear across all states of charge, with general guidance that it drops off at higher states of charge. Details concerning actual charge efficiency as a function of state-of-charge (SOC) would be very useful to PV system designers to allow informed trade-off decisions involving battery size, battery daily depth of

This work supported by the Photovoltaic Energy Technology Division of the US Department of Energy under contract DE-AC04-94AL85000. discharge and PV array size. Hence, a procedure has been developed, and is described herein, to acquire these efficiency versus SOC measurements. Preliminary results agree with existing general knowledge, and provide the details of charge efficiency versus state of charge for the specific battery under test.

Specific charge versus state of charge information is particularly important for systems where a very large battery (that is, one designed to normally operate in the upper $10 \%$ or less of state of charge in order to achieve high load availability) is used. For example, a PV system for an area light may be designed to allow the light to not function for a couple of nights per year, but a communication repeater may be only allowed a couple of hours per year of outage time (often less). One common method for increasing the availability of PV systems is to increase the size of the battery. Increasing battery size in a system implies that the battery will be operating at a higher average state-of-charge. If a 100 amp-hour (Ah) battery is used in a system with a 30Ah daily load, then one would expect the battery to be operating in the $70 \%$ to $100 \%$ SOC regime on the average. If this same load was operated with a 300Ah battery, then the battery would be expected to operate in the $90 \%$ to $100 \%$ SOC regime on the average. Because charge efficiency decreases with increasing battery state-of-charge, the system with the larger battery may also need a larger PV array to account for the higher losses associated with operating at a higher average SOC. Battery charge efficiency is also a function of charge rate, with lower rates resulting in higher efficiencies. The larger battery will be operating with a lower charge rate, which will result in higher charge efficiency. $A$ decision on increased array size must be made with full knowledge of charge efficiency at the actual charge rate being employed.

The testing reported on here examined a single sample of the Trojan 30XHS battery. This is a 12-volt, flooded, lead-antimony battery rated 130Ah at the 20 hour rate by the manufacturer. Testing in PV applications, where charging is rarely in accordance with manufacturer's recommendations, indicates that this battery has a "PV capacity" of about 100Ah, and that is the value that will be used as this battery's capacity in this paper. 


\title{
$5911096-1736 C$ CONF- $9605 / 3--18$ \\ A Study of Lead-Acid Battery Efficiency Near Top-of-Charge and the Impact on PV System Design
}

\author{
John W. Stevens and Garth P. Corey \\ Sandia National Laboratories, Photovoltaic System Applications Department \\ Sandia National Laboratories, Battery Analysis and Evaluation Department \\ PO Box 5800, MS 0753 \\ Albuquerque, New Mexico 87185-0753
}

\begin{abstract}
Knowledge of the charge efficiency of lead-acid batteries near top-of-charge is important to the design of small photovoltaic systems. In order to know how much energy is required from the photovoltaic array in order to accomplish the task of meeting load, including periodic full battery charge, a detailed knowledge of the battery charging efficiency as a function of state of charge is required, particularly in the high state-of-charge regime, as photovoltaic systems are typically designed to operate in the upper 20 to $30 \%$ of battery state-of-charge. This paper presents the results of a process for determining battery charging efficiency near top-of-charge and discusses the impact of these findings on the design of small PV systems.
\end{abstract}

\section{BACKGROUND}

Batteries are often necessary in photovoltaic (PV) systems to store energy generated while the sun is shining. Therefore, it is important to understand the specific requirements of batteries when designing a PV system. This includes an understanding of the amount of energy that will be lost in battery charging. Overestimating these battery charging losses results in a larger PV array than required, whereas underestimating them results in unanticipated loss of load as well as the possibility of damaging batteries because of lack of providing a periodic high state-of-charge.

it is generally understood that battery charge efficiency is high (above $95 \%$ ) at low states of charge and that this efficiency drops off near full charge. However, actual battery charge efficiencies are often stated as though efficiency is linear across all states of charge, with general guidance that it drops off at higher states of charge. Details concerning actual charge efficiency as a function of state-of-charge (SOC) would be very useful to PV system designers to allow informed trade-off decisions involving battery size, battery daily depth of

This work supported by the Photovoltaic Energy Technology Division of the US Department of Energy under contract DE-AC04-94AL85000. discharge and PV array size. Hence, a procedure has been developed, and is described herein, to acquire these efficiency versus SOC measurements. Preliminary results agree with existing general knowledge, and provide the details of charge efficiency versus state of charge for the specific battery under test.

Specific charge versus state of charge information is particularly important for systems where a very large battery (that is, one designed to normally operate in the upper $10 \%$ or less of state of charge in order to achieve high load availability) is used. For example, a PV system for an area light may be designed to allow the light to not function for a couple of nights per year, but a communication repeater may be only allowed a couple of hours per year of outage time (often less). One common method for increasing the availability of PV systems is to increase the size of the battery. Increasing battery size in a system implies that the battery will be operating at a higher average state-of-charge. If a 100 amp-hour (Ah) battery is used in a system with a 30Ah daily load, then one would expect the battery to be operating in the $70 \%$ to $100 \%$ SOC regime on the average. If this same load was operated with a $300 \mathrm{Ah}$ battery, then the battery would be expected to operate in the $90 \%$ to $100 \%$ SOC regime on the average. Because charge efficiency decreases with increasing battery state-of-charge, the system with the larger battery may also need a larger PV array to account for the higher losses associated with operating at a higher average SOC. Battery charge efficiency is also a function of charge rate, with lower rates resulting in higher efficiencies. The larger battery will be operating with a lower charge rate, which will result in higher charge efficiency. A decision on increased array size must be made with full knowledge of charge efficiency at the actual charge rate being employed.

The testing reported on here examined a single sample of the Trojan 30 XHS battery. This is a 12-volt, flooded, lead-antimony battery rated 130Ah at the 20 hour rate by the manufacturer. Testing in PV applications, where charging is rarely in accordance with manufacturer's recommendations, indicates that this battery has a "PV capacity" of about 100Ah, and that is the value that will be used as this battery's capacity in this paper. 


\section{DISCLAIMER}

Portions of this document may be illegible in electronic image products. Images are produced from the best available original document. 
Once this testing has been completed, and the results have been reviewed, other batteries will be tested to examine the applicability of these results to other batteries of both similar and different types.

\section{CHARGE RATE SELECTION}

For this initial test sequence, a single charge and discharge rate was selected. The rate was chosen as one of many that is typically seen in PV systems. For the 100Ah Trojan 30XHS battery, a charge and discharge rate of 3.3 amps, or $C / 30$, was selected. PV system batteries will normally have charge rates that vary from about $1 / 10$ th the battery capacity to about $1 / 50$ th the battery capacity, or $\mathrm{C} / 10$ to $\mathrm{C} / 50$, with discharge rates varying from about $\mathrm{C} / 10$ to $\mathrm{C} / 150$. A moderate rate of $\mathrm{C} / 30$ was selected from these ranges, resulting in 3.3 amps. It is important to select a charge and discharge rate that is similar to that used in PV systems because these rates will have a significant effect on battery charge efficiency,

An example demonstrates the origin of these ranges. Consider a load, including losses, that requires 15Ah per day from the PV array. In an area of a good solar resource of $5 \mathrm{kWh} / \mathrm{m}^{2} /$ day, also referred to as 5 sunhours per day, this energy will be acquired from the array over the daylight hours, with peak current of 3 amps (15 Amp-hours/5 sun-hours) occurring in the hour around noon. A typical design might require "5 days storage" for the batteries. That is, the batteries would be required to provide 75Ah to the load with no additional solar energy input. PV systems are typically designed with a "lowvoltage-disconnect" (LVD) to keep the battery from experiencing $100 \%$ discharges. A typical LVD might be designed to allow $75 \%$ discharge. This then implies that the $75 \mathrm{Ah}$ energy storage is $75 \%$ of the battery capacity, so we will be considering a 100Ah battery. Therefore, the peak charge rate will be $100 / 3=33$, or a charge rate of $\mathrm{C} / 33$ (that is, the charge rate is equal to the battery capacity divided by 33 ). A 3 sun-hour per day locale would require a larger array with a 5 amp peak, resulting in a peak charge rate of $\mathrm{C} / 20$. If the load is a light that is on all night, the discharge rate will be $15 \mathrm{Ah} / 12$ hours = 1.25 amp or $\mathrm{C} / 80$. If the load remains $15 \mathrm{Ah}$ per day, but is spread over 24 hours per day, the discharge rate will be $\mathrm{C} / 160$.

\section{TEST PROCEDURE}

All tests were performed on a Digitron/Firing Circuits BTS 600 charge/discharge test unit, which charges with pure dc (as observed on an oscilloscope.) This test unit allows programming several test sequences, then performs the tests while monitoring and logging the test data. The test equipment and battery are in an air conditioned room with the room temperature maintained near $72^{\circ} \mathrm{F}$. The battery temperature was monitored and recorded. Because of the low charge/discharge rate of the testing $(\mathrm{C} / 30)$, the battery and room temperatures remained essentially the same.
All discharges were to 10.5 volts to ensure consistency in counting amp-hours. The battery was first charged and discharged through 10 complete cycles in order to "form" the battery and ensure consistent results. Full recharge (as opposed to the partial charges used to charge the battery with a specific number of Amp-hours) was performed by bringing the battery voltage to 14.8 volts and then maintaining regulation voltage (14.8 volts) by tapering the current for 10 hours.

A test procedure was developed to charge the battery in steps beginning with approximately $65 \%$ capacity, then increasing the input in $8 \mathrm{Ah}$ increments until about 100Ah output is obtained on discharge. The Digitron tester calculates both amp-hours and watt-hours for each data point, so both Ah and energy efficiency can be easily obtained with the same set of test data.

Two types of efficiency are calculated using the test data. These will be referred to as overall average efficiency (the efficiency from zero SOC to that SOC under test) and incremental efficiency (the efficiency between two non-zero states of charge, for example, between 80 and $85 \% \mathrm{SOC}$ ). After the initial regime of 10 full charge/discharge cycles, the battery was charged with $68 \mathrm{Ah}$ which was estimated to result in about $65 \%$ SOC, or that would provide about 65Ah on discharge. The 68Ah charge actually resulted in an average discharge of 65.9Ah. After the battery was charged with 68Ah, it was then discharged to determine the amp-hours available, and charge efficiency was calculated. This procedure was repeated several times for each SOC level in the testing. The amp-hours input was then increased and the next level of SOC was examined in a similar manner.

\section{RESULTS}

The results of this testing are displayed graphically in Figure 1. Each data point in Figure 1 represents at least four tests. Some represent more than this, as some tests were repeated at random to verify repeatable results. The greatest variance in test results for each step was $5.8 \%$, with several of the steps resulting in variance in results of less than $2 \%$. The data is represented as a minimum value of amp-hours extracted for each charge level, a maximum value and the average. This graph shows that the scatter among data is relatively small.

Figure 2 shows the conversion of the amp-hours out versus amp-hours in to efficiencies. Notice that there are two curves, one displaying overall efficiency from zero state of charge to the particular state of charge under test, and the other showing incremental efficiency between states of charge. Notice also that the overall efficiency shows high values, with full charge represented by approximately $85 \%$ efficiency, a commonly used value for battery charge efficiency. More importantiy, notice the dramatically lower efficiencies for the increments above about $80 \%$ state of charge, where most values are below $60 \%$ efficiency, and full charge is represented by less than $50 \%$ efficiency. (Actually, full charge, resulting in 100Ah output has not been reached in the testing to date. The greatest output was $96.5 \mathrm{Ah}$, which resulted from 116Ah input. An attempt to achieve 100Ah output will be made as part of the conclusion of this testing.) 


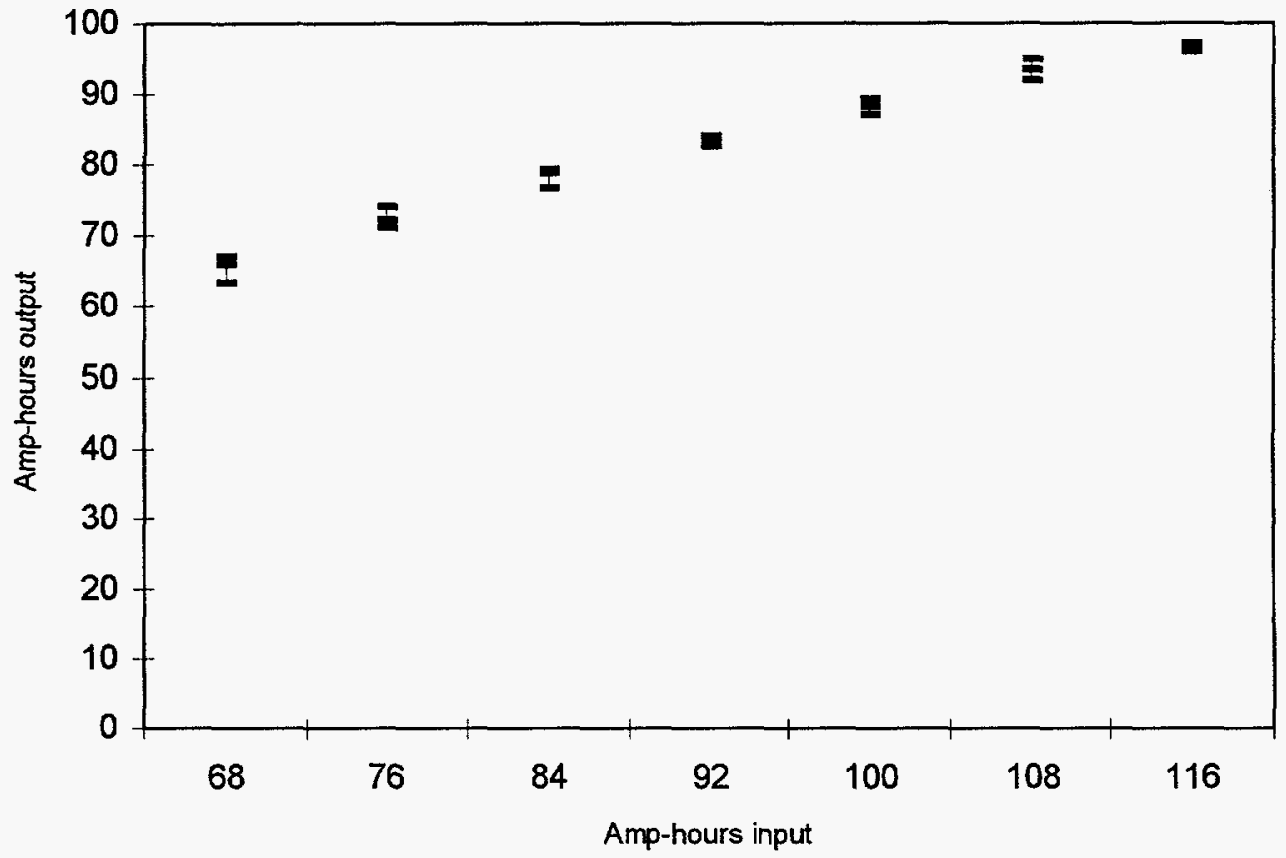

Figure 1. Minimum, maximum and average values of test data, showing small scatter in test results.

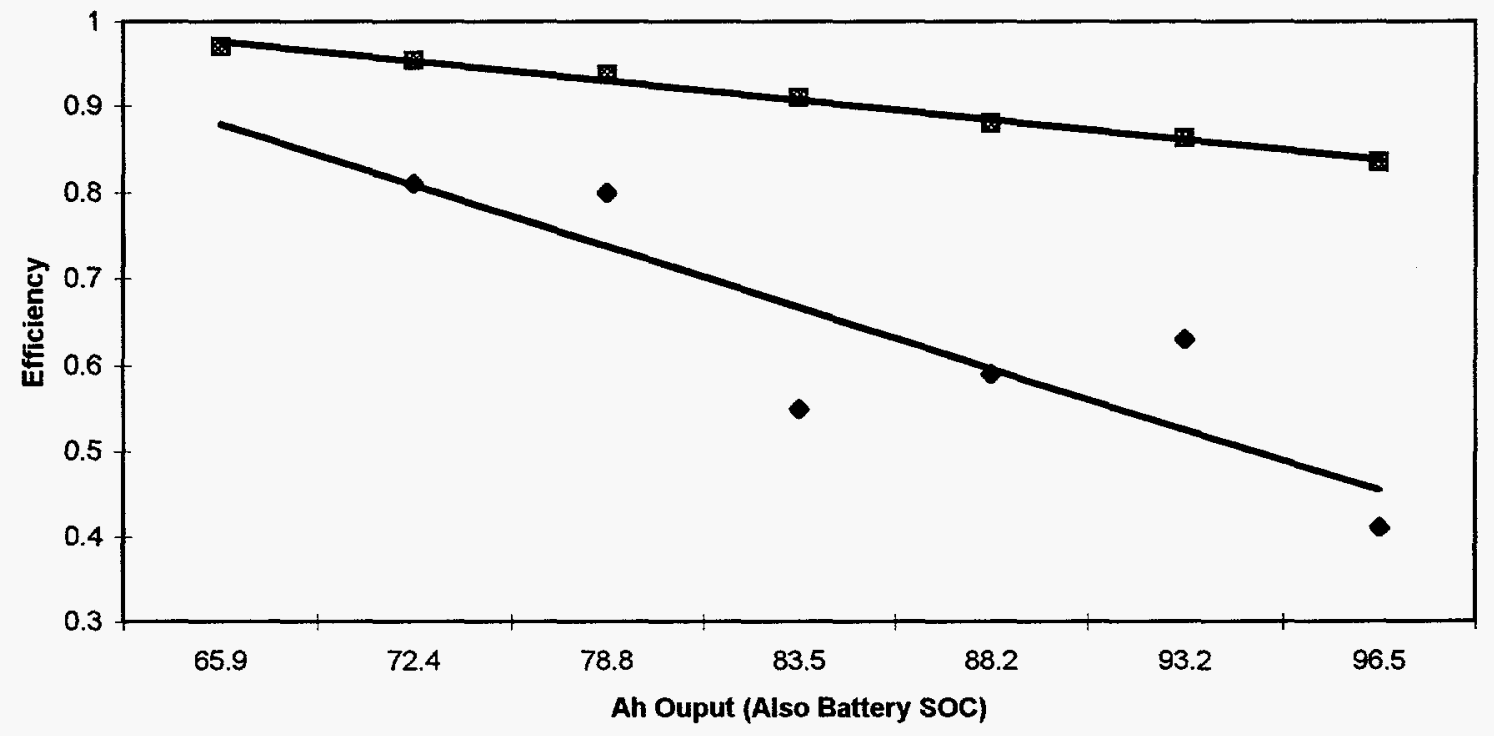

Figure 2. Incremental charge efficiency is dramatically less than overall charge efficiency at the higher states of charge.

Clearly, the use of assumed charge efficiencies in the range of $80 \%$ will not result in a fully charged battery when this battery is expected to operate in the upper $20 \%$ of it's state of charge. It is expected that these results will hold up well for other deep-cycle flooded lead-antimony batteries as well. 


\section{INTERMEDIATE FULL CHARGE CYCLES}

An observation early in the testing required a change in the test procedure. The original intent had been to perform several partial charge/discharge cycles in sequence. For example, charge to 68Ah input, discharge, then charge to 68Ah input and so on until the four complete cycles at $68 \mathrm{Ah}$ input were complete. Then fully charge and discharge the battery before proceeding with the next level. It was seen early in the testing that this was not going to work, as the capacity resulting from 68Ah input dropped with each succeeding cycle when no full charge cycles were performed between partial charge cycles. Therefore a full charge and discharge cycle was added between each partial charge/discharge cycle.

This result has important implications to operational PV systems. That is, if a battery is partially charged for several consecutive cycles (for example, the array is marginally sized and there is a series of less than full sun days in the winter) the useable battery capacity decreases each cycle, even though the same amount of energy has been presented to the battery each day. This is the result of battery inefficiencies, electrolyte stratification, and sulfate buildup during these partial charges. An associated full charge, with its attendant gassing, is needed to destratify the electrolyte and remove the residual sulfate. This sulfate buildup can become a problem if this pattern continues for several months. In the short term it can be reversed by a full "equalizing" type charge, which, in most cases is not possible in small PV systems. Battery equalization requires a PV charge controller that has been specifically designed to include this function. At low charge rates (for example, less than $\mathrm{C} / 40$ ) equalization may not be possible because of charging time limitations. In any case, this reduction in useable capacity will impact system availability and should be understood.

\section{FURTHER TESTING}

The current set of tests will be completed by decreasing the input to the battery progressively until a point is found at which the incremental charge efficiency stabilizes, implying that this value will prevail throughout the lower states of charge. During this testing, earlier tests will be repeated as a check and to investigate the continued health of the battery (that is, looking for changes in results that would indicate changing battery health.) Finally, these testing procedures will be applied to other batteries to see how common the results are and to examine variations that may be found.

\section{CONCLUSIONS}

A test procedure has been developed to allow the examination of battery charge efficiency as a function of battery state of charge. Preliminary results agree well with established general understanding that the charge efficiency of flooded lead-antimony batteries declines with increasing state-of-charge, and that charge efficiency is a non-linear function of battery state-of-charge. These tests indicate that from zero SOC to $84 \%$ SOC the average overall battery charging efficiency is $91 \%$, and that the incremental battery charging efficiency from $79 \%$ to $84 \%$ is only $55 \%$. This is particularly significant in PV systems where the designer expects the batteries to normally operate at SOC above $80 \%$, with deeper discharge only occurring during periods of extended bad weather. In such systems, the low charge efficiency at high SOC may result in a substantial reduction in actual available stored energy because nearly half the avallable energy is serving losses rather than charging the battery. Low charging efficiency can then result in the battery operating at an average $\mathrm{SOC}$ significantly lower than the system designer would anticipate without a detailed understanding of charge efficiency as a function of SOC. During normal weather, capacity degradation will not be evident, but it will manifest itself when the battery is called on to provide the full purchased capacity, which will be found to be unavailable. Extended operation in a low SOC environment can also result in permanent loss of capacity from sulfation if the battery is operated for long periods of time without a sufficient recovery or equalizing charge.

The impact of low charge efficiency at high states of charge has the greatest potential impact on systems where high energy availability is needed. Such systems usually utilize large batteries to ensure energy availability during the longest stretches of bad weather. This may not provide the energy required if the PV array is insufficient to provide a recovery charge for batteries at $90 \%$ SOC and above, where charge efficiency is very low. Charge efficiencies at $90 \%$ SOC and greater were measured at less than $50 \%$ for the battery tested here, requiring a PV array that supplies more than twice the energy that the load consumes for a full recovery charge. Many batteries in PV systems never reach a full state of charge, resulting in a slow battery capacity loss from stratification and sulfation over the life of the battery.

\section{DISCLAIMER}

\footnotetext{
This report was prepared as an account of work sponsored by an agency of the United States Government. Neither the United States Government nor any agency thereof, nor any of their employees, makes any warranty, express or implied, or assumes any legal liability or responsibility for the accuracy, completeness, or usefulness of any information, apparatus, product, or process disclosed, or represents that its use would not infringe privately owned rights. Reference herein to any specific commercial product, process, or service by trade name, trademark, manufacturer, or otherwise does not necessarily constitute or imply its endorsement, recommendation, or favoring by the United States Government or any agency thereof. The views and opinions of authors expressed herein do not necessarily state or reflect those of the United States Government or any agency thereof.
} 